\title{
'BUSINESS AS USUAL': IMPLEMENTATION OF ADVANCE CARE DIRECTIVES IN A RENAL DIALYSIS UNIT
}

A Larmer, ${ }_{1}^{1}$ T Battese, ${ }^{1}$ F Westgarth ${ }^{2}{ }^{1}$ Ballina Renal Unit, New South Wales, Australia; ${ }^{2}$ Agency for Clinical Innovation (ACI), New South Wales, Australia

\subsection{6/bmjspcare-2013-000491.57}

Background Advance care directives (ACDs) are designed to empower patients and give them a choice in end of life (EOL) care. According to the NSW Health policy established in 2004, ACDs are to be present in patients' medical records for moments when the patients are unable to make decisions for themselves. In 2009, two incidents highlighted the need to implement ACDs as a standard component of the model of care in our unit.

Aim To normalise the establishment of ACDs for patients in our Unit, and to have at least $80 \%$ of our renal dialysis patients with up-to-date and easily accessible ACD in place.

Methods Project guidance was provided to the Ballina project team via the ACI Haemodialysis Models of Care Program. The project involved staff training, patient education, development of an ACD Form, and process change within the renal unit and the hospital. The process was adaptable, flexible and responsive, and staff developed confidence in addressing this difficult issue with their patients. Furthermore, a system was developed to include ACD alerts on the patient's electronic medical record (eMR), making this information available at all access points throughout the health district.

Results ACDs have been established as a standard component of the model of dialysis care within our Unit. As a result of the project the proportion of our patients with active ACDs rose from $<5 \%$ to above $85 \%$. There remain some difficulties broaching this topic with some cultural groups.

Discussion ACDs have the ability to prevent overuse of medical resources and decrease family burden during EOL care. During education sessions it was found that staff had difficulty approaching their

patients on these very sensitive and challenging issues, and required considerable training to empower them to support their patients' during advance care planning (ACP). The successful implementation was due to the patients perceiving it as a normal process that they themselves should drive, in collaboration with their families and with their clinical carers.

Conclusion ACP policies require considerable staff training and support to enable successful implementation. 